MATHEMATICS OF COMPUTATION

Volume 65, Number 216

October 1996, Pages 1513-1530

\title{
STRICTLY POSITIVE DEFINITE FUNCTIONS ON SPHERES IN EUCLIDEAN SPACES
}

\author{
AMOS RON AND XINGPING SUN
}

\begin{abstract}
In this paper we study strictly positive definite functions on the unit sphere of the $m$-dimensional Euclidean space. Such functions can be used for solving a scattered data interpolation problem on spheres. Since positive definite functions on the sphere were already characterized by Schoenberg some fifty years ago, the issue here is to determine what kind of positive definite functions are actually strictly positive definite. The study of this problem was initiated recently by Xu and Cheney (Proc. Amer. Math. Soc. 116 (1992), 977-981), where certain sufficient conditions were derived. A new approach, which is based on a critical connection between this problem and that of multivariate polynomial interpolation on spheres, is presented here. The relevant interpolation problem is subsequently analyzed by three different complementary methods. The first is based on the de Boor-Ron general "least solution for the multivariate polynomial interpolation problem". The second, which is suitable only for $m=2$, is based on the connection between bivariate harmonic polynomials and univariate analytic polynomials, and reduces the problem to the structure of the integer zeros of bounded univariate exponentials. Finally, the last method invokes the realization of harmonic polynomials as the polynomial kernel of the Laplacian, thereby exploiting some basic relations between homogeneous ideals and their polynomial kernels.
\end{abstract}

\section{INTRODUCTION}

Let $S^{m-1}$ denote the unit sphere in the Euclidean space $\mathbb{R}^{m}(m \geq 2)$, and $d_{m}$ the geodesic distance on $S^{m-1}$, i.e.,

$$
d_{m}(x, y)=\operatorname{Arccos}(x \cdot y), \quad x, y \in S^{m-1} .
$$

Here $x \cdot y$ denotes the usual inner product of $x$ and $y$. Let $g:[0, \pi] \rightarrow \mathbb{R}$ be a continuous function, and let $\Theta \subset S^{m-1}$ be of cardinality $n$. We study in this paper the possible strict positive definiteness of the $n \times n$ matrix

$$
A:=A_{\Theta}:=A_{g, \Theta}
$$

whose rows and columns are indexed by $\Theta$, and whose $(\theta, \vartheta)$-entry is

$$
g\left(d_{m}(\theta, \vartheta)\right)
$$

Received by the editor February 7, 1994 and, in revised form, February 22, 1995 and July 5, 1995.

1991 Mathematics Subject Classification. Primary 42A82, 41A05; Secondary 33C55, 33C90.

(C)1996 American Mathematical Society 
i.e., for any arbitrary ordering $\left(\theta_{1}, \theta_{2}, \ldots\right)$ of the points in $\Theta$ we look for conditions such that

$$
c^{T} A c=\sum_{i=1}^{n} \sum_{j=1}^{n} c_{i} c_{j} g\left(d_{m}\left(\theta_{i}, \theta_{j}\right)\right)>0, \quad \forall c=\left(c_{1}, \ldots, c_{n}\right) \in \mathbb{R}^{n} \backslash 0 .
$$

The matrix $A$ of (1.1) naturally arises in the study of approximations to scattered data on spheres. Given $f$ : $\Theta$ (i.e., a function $f$ defined, at least, on the set $\Theta$ ), one may choose to interpolate $f$ on $\Theta$ by a function $g_{f}$ in the linear space

$$
G_{\Theta}:=\operatorname{span}\left\{g\left(d_{m}(\cdot, \theta)\right): \theta \in \Theta\right\},
$$

with $g:[0, \pi]$ a fixed univariate function. The existence of a unique interpolant $g_{f} \in G_{\Theta}$ for $f$ then amounts to the invertibility of the above matrix $A$. Of course, if $A$ is also positive definite, then the finding of $g_{f}$, i.e., the inversion of $A$, can then be approached by efficient and stable numerical (iterative or direct) methods.

In view of the above, the following problem becomes self-suggestive:

Problem 1.3. Determine conditions under which the interpolation matrix $A_{\Theta}$ of (1.1) is

(a) positive definite (for $S^{m-1}$ ), for any $\Theta \subset S^{m-1}$ of cardinality $n$, for some fixed $n$;

(b) positive definite (for $S^{m-1}$ ), for any $\Theta \subset S^{m-1}$;

$(\mathrm{c}, \mathrm{d})$ same as $(\mathrm{a}, \mathrm{b})$, with "invertibility" replacing "positive definiteness".

Definition 1.4. Let $g$ be a univariate continuous function defined on $[0, \pi]$. We say that $g$ is (strictly) positive definite of order $n$ for $S^{m-1}$ if for each $\Theta \subset S^{m-1}$ of cardinality $n$ the corresponding matrix $A_{\Theta}$ is (strictly) positive definite. A function $g$ that is (strictly) positive definite of all orders, is (strictly) positive definite.

The analogous problem in Euclidean spaces, i.e., when $\Theta \subset \mathbb{R}^{m}$, has been intensively studied in the literature. In [17], Schoenberg had characterized the positive definite functions of all orders for $\mathbb{R}^{m}$, and in [8], Micchelli established the invertibility of certain interpolation matrices arising from approximating scattered data in $\mathbb{R}^{m}$. Micchelli's results had led to a wealth of results (cf. e.g. [13], [14], [15], $[20]$ ), in which estimates for various norms and corresponding condition numbers of the interpolation matrix $A$ were established. We refer to the review article of Dyn [6] for more information on this subject. Schoenberg also considered the problem on the sphere. In [18] he proved the following characterization for a function $g$ to be positive definite of all orders. In this result, $P_{k}^{(\lambda)}$ denotes the $k$ th-degree Gegenbauer ("ultraspherical") polynomial associated with $\lambda$, [22, p. 81], [19, p. 148].

Result 1.5 [18]. A continuous function $g:[0, \pi] \rightarrow \mathbb{R}$ is positive definite on $S^{m-1}$ if and only if it has the form

$$
g(t)=\sum_{k=0}^{\infty} a_{k} P_{k}^{(\lambda)}(\cos t)
$$

in which $\lambda=(m-2) / 2, a_{k} \geq 0$, and $\sum a_{k} P_{k}^{(\lambda)}(1)<\infty$.

Our interest in the problem was initiated by the paper [23] of Xu and Cheney, where the following question is addressed: "find conditions on the coefficients $\left(a_{k}\right)_{k \in \mathbb{N}}$ in (1.6), under which $g$ is strictly positive definite" (either of a specific order 
or of all orders). Among various other results, it is shown in [23] that, if all the coefficients $a_{k}$ in (1.6) are positive, then the function $g$ is strictly positive definite on $S^{m-1}$. Further discussions of this problem can be found in Cheney and $\mathrm{Xu}$ [5], Light and Cheney [7], and Menegatto [11].

Our notion of "strict positive definiteness" is not the only possible one. For example, Narcowich, in [12], employs a stronger notion of positive definiteness and strict positive definiteness (on general compactly supported Riemannian manifolds), is able to characterize completely that stronger notion in terms of explicit verifiable conditions, and, in turn, recovers (for smooth enough $g$ ) the above-mentioned XuCheney result. His definition draws attention to classical definitions of positive definiteness in terms of smooth kernels. It is interesting to note the following: while Narcowich proves that his notion of positive definiteness is equivalent to the seemingly weaker notion of Schoenberg (the latter is the one that we adopt here), he does not have a similar equivalence between the notions of strict positive definiteness; in fact, he refers to that problem as "open". While we became aware of [12] only after being essentially done with the present endeavor, it is worth noting that Theorems $5.3(m=2)$ and 6.4 (any $m$ ) of the present paper provide a negative answer to Narcowich's open problem.

As this paper will show, a close relationship exists between the problem of determining strict positive definiteness and that of multivariate polynomial interpolation. This connection is discussed in $\S 3$, and allows us to find an equivalent version to the original problem in terms of polynomial interpolation on the unit sphere.

We then present three different methods for analyzing the equivalent polynomial interpolation problem. The first exploits the de Boor-Ron "least solution for the polynomial interpolation problem" (cf. [2], [3], [4]). This method yields that $g$ is strictly positive definite of order $n$ if (but not only if) the corresponding coefficients $a_{0}, a_{1}, \ldots, a_{\lfloor n / 2\rfloor}$ are positive (cf. Theorem 4.1 ; for the case $m=2$, this result was already proved in [23]). Another approach takes advantage of the connection between spherical harmonics on the circle and analytic polynomials, and allows us to characterize, for $m=2$, the strict positive definiteness of $g$ in terms of the positive integer zeros of univariate exponentials with real coefficients. Among the various results there, we mention Theorem 5.3, which shows that $g$ is strictly positive definite of order $n$ (on the circle) if the coefficients $\left(a_{k}\right)_{k}$ in its ultraspherical expansion contain $n$ consecutive positive coefficients. Finally, in $\S 6$, we choose a different tack which is suitable for any spatial dimension, and makes use of the fact that the harmonic polynomials are the polynomials in the kernel of the Laplacian. That direction utilizes some of the polynomial ideal basics, and its main results are collected in Theorems 6.3 and 6.4. These theorems imply, in particular, that $g$ is strictly positive definite if the set $K:=\left\{k \in \mathbb{Z}_{+}: a_{k}>0\right\}$ (with $\left(a_{k}\right)$ as in (1.6)) contains arbitrarily long sequences of consecutive even integers, as well as arbitrarily long sequences of consecutive odd integers.

We use in the paper the following notations and conventions. The symbol

\section{$\Pi$}

stands for the space of all polynomials in $m$ variables (where the value of $m$ should be clear from the context). The subspace of $\Pi$ that consists of the $k$ th-degree homogeneous polynomials is denoted by

$$
\Pi_{k}^{0}
$$


Also, given any $K \subset \mathbb{Z}_{+}$, we set

$$
\Pi_{K}:=\sum_{k \in K} \Pi_{k}^{0} .
$$

If $K=\{0,1,2, \ldots, k\}$, we often use $\Pi_{k}:=\Pi_{K}$, i.e., $\Pi_{k}$ is the space of all polynomials of degree $\leq k$. A parallel set of notations is used for harmonic polynomials. Here, we set

\section{$\mathcal{H}$}

for the space of all harmonic polynomials, and define

$$
\mathcal{H}_{k}^{0}:=\Pi_{k}^{0} \cap \mathcal{H}, \quad \mathcal{H}_{K}:=\Pi_{K} \cap \mathcal{H}, \quad \mathcal{H}_{k}:=\Pi_{k} \cap \mathcal{H} .
$$

Finally, an exponential in this paper is either a function of the form

$$
e_{\theta}: \mathbb{R}^{m} \rightarrow \mathbb{C}: x \mapsto e^{\theta \cdot x}, \quad \theta \in \mathbb{C}^{m},
$$

or any finite linear combination of such functions.

\section{Sets total with Respect to Discrete measures}

Let $\mathcal{H}_{K}$ be defined as at the end of the introduction. Given $g$, which is positive definite on $S^{m-1}$, we associate $g$ in this section with a certain $K \subset \mathbb{Z}_{+}$, and show that Problem 1.3 can be equivalently phrased in terms of the nonexistence of a nonzero discrete measure $\mu$ supported on $S^{m-1}$ that is orthogonal to the entire $\mathcal{H}_{K}$.

Since we are seeking conditions that characterize the strict positive definiteness of $g$, and since Schoenberg's theorem already characterizes the positive definiteness of $g$, we may assume that $g$ has the form (1.6). In turn, that allows us to write the matrix $A_{\Theta}$ as the infinite sum

$$
A_{\Theta}=\sum_{k=0}^{\infty} a_{k} A_{k}
$$

with

$$
A_{k}:=\left(P_{k}^{(\lambda)}(\theta \cdot \vartheta)\right)_{\theta, \vartheta \in \Theta} .
$$

Since each summand $A_{k}$ is positive definite (by virtue of Schoenberg's result, but also directly in view of the discussion below), this immediately shows that the ma$\operatorname{trix} A$ is more likely to be strictly positive definite, with the increase of the nonzero coefficients $\left(a_{k}\right)_{k}$ in the representation (1.6) of $g$. Also, since the matrix $A_{k}$ is positive definite, it can be written in the form $C_{k}^{T} C_{k}$. Among such factorizations of $A_{k}$, we select below a particular one, which is based on basic properties of spherical harmonics, and which will allow us to draw the connection between Problem 1.3 and polynomial interpolation on the sphere. In this course, it will be somewhat more convenient to work in a slightly more general setup: let $\mathcal{M}$ be any collection of real-valued Borel measures on the sphere $S^{m-1}$, and consider the map

$$
M_{k}: \mathcal{M} \rightarrow \mathbb{R}: \mu \mapsto \int_{S^{m-1} \times S^{m-1}} P_{k}^{(\lambda)}(x \cdot y) d \mu(x) d \mu(y) .
$$

Our quadratic form

$$
c \mapsto c^{T} A_{k} c
$$

can be easily seen to be a special case of the above setup, corresponding to the set $\mathcal{M}$ of all finite measures supported on $\Theta$. Thus, our original question can be viewed 
as a special case of the following one: given $\mathcal{M}$, what conditions on the nonnegative sequence $\left(a_{k}\right)_{k}$ guarantee that

$$
\sum_{k=0}^{\infty} a_{k} M_{k}(\mu)>0, \quad \forall \mu \in \mathcal{M} ?
$$

In fact, we will see in a moment that, always, $M_{k}(\mu) \geq 0$ (all $\mu$, all $k$ ). This, in turn, implies that the satisfaction of (2.3) depends only on the set

$$
K_{m, g}:=\left\{k \in \mathbb{Z}_{+}: a_{k}>0\right\},
$$

and not on the particular values the sequence $\left(a_{k}\right)$ assumes on $K_{m, g}$ : (2.3) holds if and only if there exists no nonzero $\mu \in \mathcal{M}$ whose transform $\mu \mapsto\left(M_{k}(\mu)\right)_{k=0}^{\infty}$ is supported in $\mathbb{Z}_{+} \backslash K_{m, g}$.

With these observations in hand, we start the actual analysis by recalling some basics on spherical harmonics. First, a spherical harmonic of degree $k$, is, by definition, the restriction to $S^{m-1}$ of a homogeneous harmonic polynomial of that degree. Second, spherical harmonics are connected to the ultraspherical polynomials via the following fact that can be found, for example, in Stein and Weiss [18, Chapter IV].

Let $\left\{Y_{1}^{(k)}, \ldots, Y_{h_{k}}^{(k)}\right\}$ be an orthonormal basis of $\mathcal{H}_{k}^{0}\left(\right.$ here, $\left.h_{k}:=\operatorname{dim} \mathcal{H}_{k}^{0}\right)$. Then, there is a positive constant $c_{k, \lambda}$ such that

$$
P_{k}^{(\lambda)}(x \cdot y)=c_{k, \lambda} \sum_{j=1}^{h_{k}} Y_{j}^{(k)}(x) Y_{j}^{(k)}(y) .
$$

Here, $\lambda=(m-2) / 2$, and, as before, $P_{k}^{(\lambda)}$ is the appropriate Gegenbauer polynomial.

We borrowed the idea of exploiting this important formula, i.e., the representation of the Gegenbauer polynomials in terms of spherical harmonics, from the paper [21] of the second-named author. Independently, that formula was also invoked by Narcowich in [12]. Now, (2.5) allows us to separate variables in (2.2). Indeed, substituting the former into the latter, we readily obtain

$$
M_{k}(\mu)=c_{\lambda, k} \sum_{j=1}^{h_{k}}\left|\int_{S^{m-1}} Y_{j}^{(k)} d \mu\right|^{2} .
$$

In words, up to a constant, the number $M_{k}(\mu)$ is the square of the $L_{2}\left(S^{m-1}\right)$-norm of the $k$ th component in the expansion of $\mu$ into spherical harmonics.

This implies that our original Problem 1.3 is a special case of the following:

Problem 2.7. Let $m$ be a positive integer, $K \subset \mathbb{Z}_{+}$, and $\mathcal{M}$ a collection of Borel measures defined on $S^{m-1}$. Determine whether $\mathcal{M} \cap \mathcal{H}_{K}^{\perp}=0$, in the sense that no nonzero $\mu \in \mathcal{M}$ satisfies

$$
\int_{S^{m-1}} f d \mu=0, \quad \forall f \in \mathcal{H}_{K}
$$

Here, $\mathcal{H}_{K}$ is defined as at the end of the introduction.

If $\mathcal{M} \cap \mathcal{H}_{K}^{\perp}=0$, we say that $K$ is total for $\mathcal{M}$. The above discussion shows that the matrix $A_{\Theta}$ of (1.1) is strictly positive definite if and only if, with $K_{m, g}$ defined as in (2.4), $K_{m, g}$ is total with respect to all measures $\mathcal{M}(\Theta)$ supported on $\Theta$. 
The development up to this point puts our problem in the right mathematical perspective, and shows its connection to basic harmonic analysis questions, but still falls significantly short of solving our problem: The verification whether $K$ is total or not with respect to the collection of discrete measures $\mathcal{M}(\Theta)$ does not seem to be straightforward! We mention in passing, however, that there are collections $\mathcal{M}$ with respect to which the above problem of totality is transparent:

Proposition 2.8. Let $\mathcal{M}$ be the space of all Borel measures supported on $S^{m-1}$. Then, the only subset of $\mathbb{Z}_{+}$which is total for $\mathcal{M}$ is $\mathbb{Z}_{+}$itself.

Proof. We choose first $\mu \in \mathcal{M}$ to be a spherical harmonic of degree $k$. Since spherical harmonics of other degrees are perpendicular to $\mu$, it follows immediately that $\mu \in \mathcal{H}_{K}^{\perp}$, unless $k \in K$. This shows that no proper subset of $\mathbb{Z}_{+}$is total for $\mathcal{M}$.

The fact that $\mathbb{Z}_{+}$itself is total for the space of all measures follows from the facts that (i) spherical harmonics are fundamental in $C\left(S^{m-1}\right)$, and (ii) $\mathcal{M}$ is the dual of $C\left(S^{m-1}\right)$.

We now rephrase our original problem in terms of the notion introduced in this section:

Modified Problem 2.9. Given a subset $K \subset \mathbb{Z}_{+}$, and $m>1$, determine whether $K$ satisfies the following:

(a) $K$ is total with respect to the collection $\mathcal{M}_{n}\left(S^{m-1}\right)$ of all measures on $S^{m-1}$ that are supported on a set of cardinality $\leq n$. We say then that $K$ is total of order $n\left(\right.$ on $\left.S^{m-1}\right)$.

(b) $K$ is total with respect to the set $\mathcal{M}_{0}\left(S^{m-1}\right)$ of all measures supported on finite subsets of $S^{m-1}$. We say then that $K$ is total of all orders $\left(\right.$ on $\left.S^{m-1}\right)$.

In the rest of the paper, we state our results mainly in terms of totality. It is, thus, worthwhile to summarize here the connection between this notion and the original notion of strict positive definiteness (cf. Problem 1.3): $g$ is strictly positive definite of order $n$ (respectively, of all orders) if and only if $K_{m, g}$ (defined in (2.4)) is total of order $n$ (respectively, total of all orders).

One should note that, a priori, the totality of $K$ of all orders should be easier to determine than the totality of a specific order: the space $\mathcal{M}_{0}\left(S^{m-1}\right)$ is linear, and the space $\mathcal{M}_{n}\left(S^{m-1}\right)$ is not.

\section{THE CONNECTION TO POLYNOMIAL INTERPOLATION}

Problem 2.9 is connected to the problem of interpolating by spherical harmonics on the sphere as follows:

Theorem 3.1. Let $\Theta$ be a finite subset of $S^{m-1}$, and $\mathcal{M}(\Theta)$ the space of all measures supported in $\Theta$. Given $K \subset \mathbb{Z}_{+}$, the following conditions are equivalent:

(a) $K$ is total for $\mathcal{M}(\Theta)$ (i.e., $\mathcal{H}_{K}^{\perp} \cap \mathcal{M}(\Theta)=0$ ).

(b) The restriction of $\mathcal{H}_{K}$ to $\Theta$ is of full dimension $\# \Theta$, i.e., every $f$ defined on $\Theta$ can be interpolated by a polynomial $p \in \mathcal{H}_{K}$.

Proof. Let $\delta_{\theta}$ be the functional of point evaluation at $\theta$. A general measure in $\mathcal{M}(\Theta)$ is of the form $\phi:=\sum_{\theta \in \Theta} c_{\theta} \delta_{\theta}$. Therefore, $\mathcal{M}(\Theta) \cap \mathcal{H}_{K}^{\perp} \neq 0$ if and only if 
there exists $\phi \neq 0$ as above such that

$$
\sum_{\theta \in \Theta} c_{\theta} p(\theta)=\phi(p)=0, \quad \forall p \in \mathcal{H}_{K} .
$$

This can happen if and only if not every data on $\Theta$ can be interpolated from $\mathcal{H}_{K}$.

In the rest of the paper, we exploit Theorem 3.1 in order to obtain sufficient conditions for the totality of $K \subset \mathbb{Z}_{+}$. As we mentioned before, three different approaches are employed, each occupying one of the remaining sections.

\section{The least solution of the polynomial interpolation Problem}

Recall that $\mathcal{M}_{n}$ denotes the set of all discrete measures supported on an $n$-subset of $S^{m-1}$ ( $m$ fixed), and that $K \subset \mathbb{Z}_{+}$is total for $\mathcal{M}_{n}$ if $\mathcal{M}_{n} \cap \mathcal{H}_{K}^{\perp}=0$ (cf. Problem 2.9). In this section, we find a minimal $k(n)$ such that $K$ is total for $\mathcal{M}_{n}$ in case $(0,1, \ldots, k(n)) \subset K$. Our main theorem of this type is the following:

Theorem 4.1. Let $K \subset \mathbb{Z}_{+}$. Then, given any $m \geq 2, K$ is total of order $n$ on $S^{m-1}$ if $\{0,1, \ldots,\lfloor n / 2\rfloor\} \subset K$.

Thus, our original matrix $A_{\Theta}$ is strictly positive definite whenever the first $\lfloor n / 2\rfloor+1$ coefficients in the ultraspherical expansion of $g$ are positive. For $m=2$, that result is due to [23]. The statement of the theorem is sharp in the sense that the set $K:=\{0,1, \ldots, \mid n / 2\rfloor-1\}$ is not total for $\mathcal{M}_{n}$. However, the measures in $\mathcal{M}_{n}$ that are perpendicular to $\mathcal{H}_{\lfloor n / 2\rfloor-1}$ are of a specific nature: essentially, they are supported on circles. The precise statement is as follows:

Corollary 4.2. Let $n$ be a positive integer, and let $K$ be a subset of $\mathbb{Z}_{+}$that contains the integers $\{0,1, \ldots,\lfloor n / 2\rfloor-1\}$. Further, let $\Theta$ be a subset of $S^{m-1}$ of cardinality $n$, and let $\mu \neq 0$ be a measure supported on $\Theta$. Then $\mu \in \mathcal{H}_{K}^{\perp}$ only if $\Theta$ satisfies the following:

(a) for an even $n$, $\Theta$ lies on a circle;

(b) for an odd $n \neq 5, \Theta \backslash \theta$ lies on a circle, for some $\theta \in \Theta$.

Thus, if $\Theta$ does not have the special configuration specified in the above corollary, our matrix $A_{\Theta}$ is strictly positive definite even when only the first $\lfloor n / 2\rfloor$ coefficients (in the expansion of $g$ ) are positive.

The proofs of Theorem 4.1 and Corollary 4.2, together with necessary background on polynomial interpolation, occupy the rest of this section.

Given any polynomial of degree $k$, we denote by $p_{\uparrow}$ the unique polynomial in $\Pi_{k}^{0}$ that satisfies $\operatorname{deg}\left(p-p_{\uparrow}\right)<k$, and refer to $p_{\uparrow}$ as the leading term of $p$. Also, given $p \in \Pi$, we use $p(D)$ to denote the corresponding constant-coefficient differential operator. Directional derivatives are denoted by $D_{x}$, where $x \in \mathbb{R}^{m}$ is the direction.

Given any finite $\Theta \subset \mathbb{R}^{m}$, the paper [2] introduces a polynomial space $\Pi_{\Theta}$ that satisfies all the following properties:

(a) For any function $f$ defined on $\Theta$, there exists a unique $p \in \Pi_{\Theta}$ that agrees with $f$ on $\Theta$.

(b) $\Pi_{\Theta}$ is $D$-invariant, i.e., for every $x \in \mathbb{R}^{m} \backslash 0, D_{x} \Pi_{\Theta} \subset \Pi_{\Theta}$ (equivalently, $\Pi_{\Theta}$ is translation-invariant). 
(c) $\Pi_{\Theta}$ is homogeneous. That is,

$$
\Pi_{\Theta}=\sum_{j=0}^{\infty} \Pi_{\Theta, j}
$$

where $\Pi_{\Theta, j}:=\Pi_{\Theta} \cap \Pi_{j}^{0}$.

(d) If the polynomial $p$ vanishes on $\Theta$, then its leading term $p_{\uparrow}$ annihilates $\Pi_{\Theta}$ in the sense that $p_{\uparrow}(D) \Pi_{\Theta}=0$.

(e) Conversely, every homogeneous polynomial that annihilates $\Pi_{\Theta}$ is the leading term of some other polynomial that vanishes on $\Theta$.

We refer to [2] and [4] for more details about $\Pi_{\Theta}$. The exact definition of $\Pi_{\Theta}$ will be given in the sequel; for the time being, though, we need only the fact that a polynomial space that satisfies these five properties exists.

Lemma 4.3. Let $k$ be the least integer that satisfies $\Pi_{\Theta} \subset \Pi_{k}$. If $\Theta \subset S^{m-1}$, then

$$
\operatorname{dim} \Pi_{\Theta, j} \geq 2, \quad j=1,2, \ldots, k-1 .
$$

Proof. We assume that the claim of the lemma is false, and will seek a contradiction. Let $0<j<k$ be the maximal integer that violates the lemma's claim. If $j=k-1$, then $\Pi_{\Theta, j+1}=\Pi_{\Theta, k} \neq 0$, by the definition of $k$. Otherwise, by the maximality of $j$, $\operatorname{dim} \Pi_{\Theta, j+1} \geq 2$. Either way, $\Pi_{\Theta, j+1}$ contains a nonzero polynomial $p$. We consider the map $\psi: \mathbb{R}^{m} \rightarrow \Pi_{j}^{0}$ defined by

$$
\psi(x)=D_{x} p .
$$

Since $\Pi_{\Theta}$ is $D$-invariant, $\operatorname{ran} \psi \subset \Pi_{\Theta}$, hence $\operatorname{ran} \psi \subset \Pi_{\Theta, j}$. Since $\operatorname{deg} p>0$, we have $\psi \neq 0$. On the other hand, $\operatorname{dim} \operatorname{ran} \psi \leq \operatorname{dim} \Pi_{\Theta, j} \leq 1$. Therefore, $\operatorname{dim} \operatorname{ran} \psi=1$, hence $\operatorname{dim} \operatorname{ker} \psi=m-1$. Since $\psi$ is linear, its kernel, then, is a hyperplane, and $p$ has to be a univariate polynomial of the form

$$
p(y)=c(\xi \cdot y)^{j+1},
$$

with $\xi$ perpendicular to the above hyperplane.

Let $\delta(D)$ be the $m$-dimensional Laplacian. We note that, since $j \geq 1, \delta(D) p=$ $c^{\prime} \sum_{i=1}^{m} \xi_{i}^{2}(\xi \cdot y)^{j-1} \neq 0$. On the other hand, the quadratic polynomial $q:=\delta-1$ vanishes on $\Theta$, hence by property (d) of $\Pi_{\Theta}, \delta(D)=q_{\uparrow}(D)$ annihilates the entire $\Pi_{\Theta}$. In particular, $\delta(D) p=0$, which contradicts the previous conclusion.

Lemma 4.4. For any $\Theta \subset S^{m-1}$ of cardinality $n$, the following is true:

(a) There exists a space $\Pi_{\Theta} \subset \mathcal{H}_{\lfloor n / 2\rfloor}$ such that interpolation from $\Pi_{\Theta}$ to any $f$ defined on $\Theta$ is always possible, i.e., the restriction of $\Pi_{\Theta}$ to $\Theta$ is of dimension $n=\# \Theta$.

(b) If $n$ is even, and $\Theta$ does not lie on a circle, then $\Pi_{\Theta} \subset \mathcal{H}_{n / 2-1}=\mathcal{H}_{\lfloor n / 2\rfloor-1}$.

(c) If $n>5$ is odd and no $(n-1)$ points of $\Theta$ lie on a circle, then $\Pi_{\Theta} \subset \mathcal{H}_{(n-3) / 2}=$ $\mathcal{H}_{\lfloor n / 2\rfloor-1}$.

Proof. Claim (a) of Lemma 4.4 follows from Lemma 4.3. With $\Pi_{\Theta}$ the least space of [2], the proof of Lemma 4.3 shows that $\Pi_{\Theta}$ is annihilated by the Laplacian, hence consists of harmonic polynomials, whenever $\Theta \subset S^{m-1}$. Then, since $\Pi_{\Theta}$ is translation-invariant, it must contain the constants, hence $\operatorname{dim} \Pi_{\Theta, 0}=1$. Also, by the definition of $k, \operatorname{dim} \Pi_{\Theta, k} \geq 1$. Hence, Lemma 4.3 implies that

$$
\# \Theta=\operatorname{dim} \Pi_{\Theta} \geq 1+2(k-1)+1=2 k,
$$


with the right-most equality due to property (a) of $\Pi_{\Theta}$.

The proof of (b) in Lemma 4.4 is also quite simple. We present, however, a slightly longer proof, which prepares also for the proof of (c). We assume that $n$ is even, and that $\Pi_{\Theta} \not \subset \Pi_{n / 2-1}$, and will prove that $\Theta$ lies on a circle. First, by (a) of Lemma 4.4, $\Pi_{\Theta} \subset \Pi_{n / 2}$. Second, we have $\operatorname{dim} \Pi_{\Theta, 0}=1$, and, since we assume that $\operatorname{dim} \Pi_{\Theta, n / 2} \geq 1$, Lemma 4.4 implies that $\operatorname{dim} \Pi_{\Theta, j} \geq 2, j=1,2, \ldots, n / 2-1$. Taking into account the fact that $\Pi_{\Theta}$ is $n$-dimensional, we realize that none of these inequalities can be sharp, i.e., the homogeneous dimensions of $\Pi_{\Theta}$ are

$$
1,2,2, \ldots, 2,1 \text {. }
$$

Let $q \in \Pi_{\Theta, j} \backslash 0,1<j \leq n / 2$, and let $\psi_{q}$ be the corresponding map that was introduced in the proof of Lemma 4.4. The proof of that lemma shows that $\operatorname{ran} \psi_{q} \subset$ $\Pi_{\Theta, j-1}$. Since $\operatorname{dim} \Pi_{\Theta, j-1}=2$, this implies that $\operatorname{dim} \operatorname{ker} \psi_{q} \geq m-2$, i.e., that $q$ is a bivariate polynomial. Further, the argument in Lemma 4.4 makes clear that $q$ cannot be univariate. Consequently, $\operatorname{rank} \psi_{q}=2$, and $\operatorname{ran} \psi_{q}=\Pi_{\Theta, j-1}$. By selecting $p$ to be any nonzero polynomial in $\Pi_{\Theta, n / 2}$, and choosing $\alpha \in \mathbb{Z}_{+}^{d}$ such that $|\alpha|=n / 2-j$ and $q:=D^{\alpha} p \neq 0$, we obtain that each $\Pi_{\Theta, j-1}, j=2, \ldots, n / 2$, is generated by derivatives of $p$. That same result is trivial for $j=n / 2+1$ (since $\Pi_{\Theta, n / 2}$ is 1 -dimensional), and for $j=1$. Thus,

$$
\Pi_{\Theta}=\{P(D) p: P \in \Pi\},
$$

i.e., $\Pi_{\Theta}$ comprises the derivatives of the single polynomial $p$, hence, in particular, $\Pi_{\Theta}$ is annihilated by the $(m-2)$-dimensional space $\left\{D_{x}: x \in \operatorname{ker} \psi_{p}\right\}$. Selecting any basis for that space, we invoke property (e) of $\Pi_{\Theta}$ to conclude that there exist $m-2$ linearly independent linear polynomials, each of which vanishes on $\Theta$, i.e., that $\Theta$ lies on a 2-dimensional linear manifold. Since $\Theta$ is assumed to lie also on $S^{m-1}$, we conclude that, indeed, it lies on a circle. This completes the proof of (b).

Before we prove (c), we mention that its statement is sharp in the following sense. First, if $\Theta$ consists of five points in $S^{2}$, then, regardless of its distribution, the 5dimensional $\Pi_{\Theta}$ cannot lie in the 4-dimensional $\Pi_{(n-3) / 2}=\Pi_{1}$, i.e., the statement in (c) fails to hold for $n=5$. Further, if all the points of $\Theta$ except one lie on a circle, then $\Pi_{\Theta} \not \subset \Pi_{(n-3) / 2}$, and, therefore, one cannot prove in (c) that $\Theta$ must lie entirely on a circle.

In order to prove (c), we assume that $n \geq 7$ is odd, and that $\Pi_{\Theta} \not \subset \Pi_{(n-3) / 2}$. We need to prove then that, save perhaps for one point, $\Theta$ lies on a circle. Here, we need to recall from [2] that the definition of $\Pi_{\Theta}$ is

$$
\Pi_{\Theta}=\operatorname{span}\left\{f_{\downarrow}: f \in E(\Theta)\right\},
$$

with $E(\Theta)$ the span of the exponentials $e_{\theta}, \theta \in \Theta$, and with $f_{\downarrow}$ the smallestdegree nonzero homogeneous term in the power expansion of $f$. It is then easy to check that, if $\Theta$ contains four points which are not co-planar, then $\operatorname{dim} \Pi_{\Theta, 1} \geq 3$. Therefore, assuming $\Theta$ not to lie on a circle, we must have that $\operatorname{dim} \Pi_{\Theta, 1} \geq 3$. Repeating the same counting arguments that we employed in the proof of (b), we conclude that the homogeneous dimensions of $\Pi_{\Theta}$ must be

$$
1,3,2,2, \ldots, 2,1 \text {. }
$$

Selecting any $p \in \Pi_{\Theta,(n-1) / 2}$, and repeating the argument that was used in the proof of (b), we conclude that $p$ is bivariate and that the derivatives of $p$ form a subspace in $\Pi_{\Theta}$ of dimension $n-1$ (the argument relies on the fact that $\operatorname{dim} \Pi_{\Theta,(n-1) / 2-1}=2$, hence requires $n \geq 7)$. Let $x \in \mathbb{R}^{m}$ be such that $D_{x} p=0$. Let also $f \in E(\Theta)$ be an 
exponential that satisfies $f_{\downarrow}=p$. Set $g:=D_{x} f, k:=(n-1) / 2$. In what follows, we denote by $f_{j}$ the $j$ th-degree homogeneous term in the power expansion of the exponential $f$. Since $f_{j}=0$, for all $j<k$, we have that $g_{j}=0$ for all $j<k-1$. Also, since $f_{k}=p$, we have that $g_{k-1}=\left(D_{x} f\right)_{k-1}=D_{x} f_{k}=D_{x} p=0$. Thus, $\operatorname{deg}\left(g_{\downarrow}\right) \geq k$. Since $g_{\downarrow}$ is in $\Pi_{\Theta}$, and $\Pi_{\Theta} \subset \Pi_{k}$, we must thus have $\operatorname{deg}\left(g_{\downarrow}\right)=k$, hence that $g_{\downarrow}$ is a constant multiple $c p$ of $p$ (since the $k$ th-degree homogeneous component of $\Pi_{\Theta}$ is spanned by $p$ ). This implies, with $q$ the linear polynomial $q(y):=x \cdot y-c$, that $(q(D) f)_{j}=0,0 \leq j \leq k$, and thereby that, if $q(D) f \neq 0$, then $\operatorname{deg}(q(D) f)_{\downarrow}>k$, in contradiction with the fact that $\Pi_{\Theta} \subset \Pi_{k}$. Thus, $q(D) f=0$.

On the other hand, as any function in $E(\Theta), f$ can be written in the form $f=$ $\sum_{\theta \in \Theta} c_{\theta} e_{\theta}$, hence $0=q(D) f=\sum_{\theta \in \Theta} q(\theta) c_{\theta} e_{\theta}$. Since finitely many exponentials are always linearly independent, we conclude that

$$
c_{\theta} \neq 0 \quad \Longrightarrow \quad q(\theta)=0
$$

Thus, the subset

$$
\Theta^{\prime}:=\left\{\theta \in \Theta: c_{\theta} \neq 0\right\}
$$

lies in the hyperplane $q=0$. Ranging the directional derivative $D_{x}$ over an $(m-2)$ dimensional space (which is possible since $p$ is bivariate), we obtain, as in the proof of (b), that $\Theta^{\prime}$ lies on a circle. It remains to show that $\# \Theta^{\prime} \geq n-1$ : since $f \in E\left(\Theta^{\prime}\right)$, and $p=f_{\downarrow}$, we have $p \in \Pi_{\Theta^{\prime}}$, and therefore its space of derivatives $D(p)$ lies in $\Pi_{\Theta^{\prime}}$ as well. Since $\operatorname{dim} D(p)$ was shown to be $n-1$, we conclude that $\# \Theta^{\prime}=\operatorname{dim} \Pi_{\Theta^{\prime}} \geq \operatorname{dim} D(p)=n-1$.

Theorem 4.1 and Corollary 4.2 follow now, in view of Lemma 4.4, from Theorem 3.1 .

\section{Sets that are total for measures on $S^{1}$ : AN ANAlytic approach}

In the case of interpolating on the circle, $\operatorname{dim} \mathcal{H}_{k}^{0}=2$ for all $k=1,2, \ldots$, and $\mathcal{H}_{k}^{0}$ is spanned by the two functions $\cos k \tau$ and $\sin k \tau$, where $(r, \tau)$ are the polar coordinates in $\mathbb{R}^{2}$. These well-known facts can be nicely used in the course of study of Problem 2.9. We will connect our problem to the distribution of zeros of bounded univariate exponentials, and use the obtained characterization to derive separate necessary and sufficient conditions for the totality of $K$ for finitely supported Borel measures.

Recall that, given $\tau \in \mathbb{C}, e_{\tau}$ is the exponential $x \mapsto e^{\tau x}$ with frequency $\tau$. Throughout the section, we will make essential use of the following univariate exponential space:

$$
\mathcal{E}_{n}:=\left\{\sum_{\tau \in \mathrm{T} \subset[0,2 \pi)} c_{\tau} e_{i \tau}: \# \mathrm{~T} \leq n, \quad c_{\tau} \in \mathbb{R}, \forall \tau\right\} .
$$

Also, $\mathcal{E}:=\bigcup_{n \geq 1} \mathcal{E}_{n}$. It is important to note that we allow only real coefficients $c_{\tau}$ in the definition.

We first record and prove the basic observation that will be utilized throughout this section.

Theorem 5.1. For $K \subset \mathbb{Z}_{+}$, the following conditions are equivalent:

(a) $K$ is total of order $n$ on $S^{1}$.

(b) $K$ is a uniqueness set for $\mathcal{E}_{n}$, i.e., only the zero function in $\mathcal{E}_{n}$ vanishes identically on $K$. 
Proof. On the circle, the homogeneous harmonic polynomial space $\mathcal{H}_{k}^{0}$ is spanned by $\sin (k \tau)$ and $\cos (k \tau)$. This means that a real-valued Borel measure $\mu$ is perpendicular to $\mathcal{H}_{k}^{0}$ if and only if

$$
\int_{S^{1}} e^{-i k \tau} d \mu(\tau)=0
$$

i.e., if and only if the Fourier transform $\widehat{\mu}$ of $\mu$ vanishes at $k$. Consequently, for any $K \subset \mathbb{Z}_{+}$, we have $\mu \in \mathcal{H}_{K}^{\perp}$ if and only if $\widehat{\mu}$ vanishes on $K$.

The assertion of the theorem then follows from the observation that $\mathcal{E}_{n}$ is the collection of all Fourier transforms of $\mathcal{M}_{n}\left(S^{1}\right)$.

The next theorem is a necessary condition for the totality of $K$ for $\mathcal{M}_{0}\left(S^{1}\right)$, and is followed by another theorem which establishes a sufficient condition for the totality of $K$ for $\mathcal{M}_{n}\left(S^{1}\right)$. Both results are based on Theorem 5.1. A result similar to the first one was mentioned by Menegatto in [9], and [10]. We note that Menegatto was then primarily interested in identifying strictly positive definite functions of all orders.

Corollary 5.2. The set $K \subset \mathbb{Z}_{+}$is total of all orders on $S^{1}$ only if it has infinite intersection with any set of the form $k \mathbb{Z}_{+}, k \in \mathbb{N}$. The same applies to sets of the form $k / 2+k \mathbb{Z}_{+}$, provided that $k$ is even.

Discussion. The above result is invalid with respect to any other arithmetic progression: one can show that the set $\mathbb{Z}_{+} \backslash\left(\alpha+k \mathbb{Z}_{+}\right)$is total of all orders, whenever $\alpha \notin \frac{k}{2} \mathbb{Z}_{+}$. As an illustration, the following observations can be made with respect to the value $k=4$ : in order to be total for $\mathcal{M}_{0}\left(S^{1}\right), K$ must have infinite intersection with $4 \mathbb{Z}_{+}$, as well as with $2+4 \mathbb{Z}_{+}$. It also must have an infinite intersection with one of the sets $1+4 \mathbb{Z}_{+}, 3+4 \mathbb{Z}_{+}$(since their union is $1+2 \mathbb{Z}_{+}$), but may have an empty intersection with the other of these latter sets.

Proof. In order to unify the proof, we assume that the relevant arithmetic progression is of the form $Z:=\alpha+k \mathbb{Z}_{+}, \alpha<k$. We will further assume that $K$ has only finite intersection with $Z$, and will use that to construct a linear combination $f^{*}$ of exponentials $\left(e_{i \tau}\right)_{\tau \in[0,2 \pi)}$ that vanishes on $K$. The crux of the proof is that, if $\alpha=0$ or $\alpha=k / 2$, the coefficients in the representation $f^{*}=\sum_{\tau} c_{\tau} e_{i \tau}$ are real. Thus, for these cases, $f^{*} \in \mathcal{E}$, and the desired result is then implied by Theorem 5.1 .

Set

$$
\mathrm{T}:=\{(2 \pi l) / k: l=0,1, \ldots, k-1\},
$$

and define the univariate exponential

$$
f:=\sum_{\tau \in \mathrm{T}} e_{i \tau}(\cdot-\alpha)
$$

Then $f(l)=0$, for $0 \leq l \leq k-1, l \neq \alpha$, and since $f$ is $k$-periodic, we conclude that $f$ actually vanishes on $\mathbb{Z}_{+} \backslash Z$.

By our assumption, the set $K \cap Z$ is finite. Let $n$ denote its cardinality, and let $t_{0}, t_{1}, t_{2}, \ldots, t_{2 n}$ be chosen in a way that (a) each set $t_{l}+\mathrm{T}$ is a subset of $[0,2 \pi)$, and (b) the sets $t_{l}+\mathrm{T}, l=0, \ldots, 2 n$, are pairwise disjoint. The restriction to $K \cap Z$ of the $2 n+1$ exponentials $\left\{e_{i t_{l}} f\right\}_{l=0}^{2 n}$ must be linearly dependent over the reals, since the set $K \cap Z$ contains no more than $n$ points. Let $f^{*}$ be a nontrivial linear combination with real coefficients of these functions that vanishes on $K \cap Z$. Furthermore, since 
$f$ vanishes on $\mathbb{Z}_{+} \backslash Z$, so does every $e_{i t_{l}} f$, and therefore $f^{*}$ vanishes on that set as well. This implies that $f^{*}$ vanishes on $K$, too. Finally, since the spectra of the exponentials $\left\{e_{i t_{l}} f\right\}_{l}$ are pairwise disjoint, $f^{*}$ cannot be identically 0 (since finitely many exponentials are linearly independent).

It remains to show that $f^{*} \in \mathcal{E}$, and this is the part where we need the assumption $\alpha=0, k / 2$. Indeed, for such choice of $\alpha$, we observe that the numbers $e_{i \tau}(-\alpha)$, $\tau \in \mathrm{T}$, are real, hence, in these cases, $f \in \mathcal{E}$, implying that $e_{i t_{l}} f \in \mathcal{E}$, too. Since $\mathcal{E}$ is a linear space over the reals, we conclude that $f^{*}$ lies in that space, too.

Consequently, we have found a nontrivial exponential $f^{*} \in \mathcal{E}$, that vanishes on $K$.

Theorem 5.3. Let $K \subset \mathbb{Z}_{+}$be given, and let $n$ be a positive integer. Suppose that one of the following two conditions holds:

(a) $K$ contains $n$ consecutive integers.

(b) $K$ contains $n$ arithmetic progressions, each of length $n$ :

$$
\alpha_{j}, \alpha_{j}+d_{j}, \ldots, \alpha_{j}+(n-1) d_{j}, \quad j=1,2, \ldots, n,
$$

and the numbers $\left(d_{1}, \ldots, d_{n}\right)$ are pairwise relatively prime.

Then $K$ is total of order $n$ on $S^{1}$.

Remark. The theorem lists two special cases of a slightly more general result (cf. $[16])$.

Proof. The proof of (a) is quite simple. We assume that there exists an exponential $f \in \mathcal{E}_{n}$ that vanishes on $K$, and will reach a contradiction. Our claim would then follow from Theorem 5.1. Being in $\mathcal{E}_{n}$, the exponential $f$ has the form

$$
f=\sum_{\tau \in \mathrm{T}} c_{\tau} e_{i \tau}, \quad \mathrm{T} \subset[0,2 \pi), c_{\tau} \in \mathbb{R} \backslash 0,
$$

with $\# \mathrm{~T} \leq n$. Without loss, we may assume that $0 \in \mathrm{T}$, and that $c_{0}=1$ (otherwise, we divide $f$ by $c_{\tau_{0}} e_{i \tau_{0}}$, with $\tau_{0}$ the smallest number in T). Let $p$ be a univariate polynomial that vanishes on $e_{i \tau}(1)=e^{i \tau}, \tau \in T \backslash 0$, and nowhere else. In particular, since $T \backslash 0 \subset(0,2 \pi), p(1) \neq 0$.

With $p(t)=\sum_{j} a_{j} t^{j}$, let $p(\nabla)$ be the induced difference operator

$$
p(\nabla): g \mapsto \sum_{j} a_{j} g(\cdot+j) .
$$

Then, $p(\nabla) e_{i \tau}=e_{i \tau} p\left(e_{i \tau}(1)\right)=0$, for each $\tau \in \mathrm{T} \backslash 0$. Hence $p(\nabla) f=p(1) \neq 0$, i.e., $p(\nabla) f$ is a nonzero constant. However, since we assume $f$ to vanish at $n$ consecutive integers, and since $p$ is of degree $<n$, it follows that $p(\nabla) f$ must vanish at least at one integer point. Contradiction. This proves (a).

The proof of (b) follows the same lines, but is more complicated. It first requires the following simple lemma, whose proof is omitted.

Lemma 5.5. Let $\mathrm{T}$ be any finite subset of $(0,2 \pi)$. Then the set of integer zeros of the function $F:=\prod_{\tau \in \mathrm{T}}\left(e_{i \tau}-1\right)$ is of the form $J \mathbb{Z}$, with $J$ a subset of $\mathbb{N} \backslash 1$ of cardinality $\leq \# \mathrm{~T}$.

The proof of (b) starts as in (a): we let $f$ be an exponential as in (5.4) that vanishes on $K$, and will show that this contradicts the assumption made on $K$. 
Again, we assume without loss that $0 \in \mathrm{T}$, and $c_{0}=1$. By Lemma 5.5, the positive integer zeros of the function

$$
F:=\prod_{\tau \in \mathrm{T} \backslash 0}\left(e_{i \tau}-1\right)
$$

are of the form $J \mathbb{N}$, with $J \subset \mathbb{N} \backslash 1$ of cardinality $<n$. This means that $F$ cannot vanish at all the numbers $d_{j}, j=1, \ldots, n$ (the relative primality assumption implies that no $k \mathbb{Z}_{+}$can contain more than one $\left.d_{j}\right)$. Without loss, we assume that $F$ does not vanish at $d:=d_{1}$, i.e., $1 \notin\left\{e_{i \tau}(d)\right\}_{\tau \in T \backslash 0}$.

Let $p$ be a polynomial

$$
p: t \mapsto \sum_{j} a_{j} t^{j}
$$

whose zero set is $\left\{e_{i \tau}(d): \tau \in \mathrm{T} \backslash 0\right\}$. Then, $\operatorname{deg} p<n$, and $p(1) \neq 0$. Let $p(\nabla)$ be the induced difference operator

$$
p(\nabla): g \mapsto \sum_{j} a_{j} g(\cdot+j d) .
$$

Then, $p(\nabla) e_{i \tau}=e_{i \tau} p\left(e_{i \tau}(d)\right)=0$, for each $\tau \in \mathrm{T} \backslash 0$. Hence $p(\nabla) f=p(1) \neq 0$, i.e., $p(\nabla) f$ is a nonzero constant. On the other hand, since $f$ is assumed to vanish on an arithmetic progression of length $n$ and stepsize $d, p(\nabla) f$ must vanish at some point, and we have thus reached the desired contradiction.

\section{Sets that are total for measures on $S^{m-1}$ : AN AlgEBraic Approach}

Here, we attack the problem from a completely different angle. While the core of the argument used in the previous section was the connection between 2-dimensional harmonic polynomials and their analytic completions, the course here is of algebraic nature. It is based on the realization of harmonic polynomials as the kernel of the Laplacian and thereby exploits the connection between a homogeneous polynomial ideal and its kernel in $\Pi$. Some of the results in this section can be, hence are, developed in a more general (and in our opinion more natural) setup.

We will require here some additional notations. The first is that of exponential spaces. These are defined in terms of some set $\Omega \subset \mathbb{R}^{m}$, and a positive integer $n$ as follows:

$$
E_{n}(\Omega):=\left\{\sum_{\theta \in \Theta} c_{\theta} e_{\theta}: \Theta \subset \Omega, \# \Theta \leq n, c_{\theta} \in \mathbb{C}, \forall \theta\right\} .
$$

(Note that $c_{\theta}$ is a constant, but $e_{\theta}$ is a function.) Obviously, the above exponential spaces are not linear spaces, in contrast with the larger space

$$
E(\Omega):=\bigcup_{n \geq 1} E_{n}(\Omega) .
$$

Another set of notations concerns maps defined on the algebra $\mathbf{A}$ of all formal power series in $m$ variables. Since $\mathbf{A}$ is the direct (infinite) sum $\sum_{k>0} \Pi_{k}^{0}$ of the homogeneous polynomial spaces, there exists, for every $k \geq 0$, a well-defined projector

$$
\bar{k}: \mathbf{A} \rightarrow \Pi_{k}^{0}
$$


that assigns to each power series $f \in \mathbf{A}$ the $k$ th-degree homogeneous component in its power expansion. Further, given a subset $K \subset \mathbb{Z}_{+}$, the sum

$$
\bar{K}:=\sum_{k \in K} \bar{k}
$$

defines an analogous projector, this time from $\mathbf{A}$ onto $\Pi_{K}$.

Finally, we reserve a special notation $\delta$ for the polynomial

$$
\delta(x):=\delta_{m}(x):=\sum_{n=1}^{m} x_{n}^{2}
$$

Thus, $\delta \mathbf{A}$ is the (homogeneous principal) ideal generated by $\delta$. Note that the evaluation $\delta(D)$ of $\delta$ at $D$ is the Laplacian operator.

Recall that a subspace $H$ of $\mathbf{A}$ or $\Pi$ is homogeneous if, for each $k \in \mathbb{Z}_{+}$, the corresponding projector $\bar{k}$ maps $H$ into itself.

Definition 6.1. Let $\Omega$ be a subset of $\mathbb{R}^{m}, H$ a homogeneous subspace of $\Pi$, and $n$ a positive integer. We say that $H$ is total of order $n$ on $\Omega$ if for any $\Theta \subset \Omega$ of cardinality $n$, and any function $F$ defined on $\Theta$, there exists a polynomial $p \in H$ that interpolates $F$ (on $\Theta$ ).

To see the connection between the new definition and our original notion of totality, one chooses $\Omega:=S^{m-1}$, and $H:=\mathcal{H}_{K}$ (defined as in the introduction). Then, in view of Theorem 3.1, the totality of $K$ of order $n$ on $S^{m-1}$ is equivalent, in terms of the new definition, to the totality of order $n$ of $\mathcal{H}_{K}$ on $S^{m-1}$.

Theorem 6.2. Let $H, n$ and $\Omega$ be as in the above definition. Then $H$ is total of order $n$ on $\Omega$ if and only if for every exponential $f \in E_{n}(\Omega)$, there exists $k \in$ $\mathbb{Z}_{+}$such that the kth-order homogeneous differential operator $\bar{k}(f)(D)$ does not annihilate $H \cap \Pi_{k}^{0}$.

Proof. For $\theta \in \mathbb{R}^{m}$, let $\delta_{\theta}$ be the functional $\delta_{\theta}: f \mapsto f(\theta)$, and for a finite set $\Theta \subset \mathbb{R}^{m}$, let $\mathcal{M}(\Theta)$ be $\operatorname{span}\left\{\delta_{\theta}\right\}_{\theta \in \Theta}$. Then $H$ fails to be total of order $n$ on $\Omega$ if and only if for some $\Theta \subset \Omega$ of cardinality $n, \operatorname{dim} H_{\mid \Theta}<\operatorname{dim} \mathcal{M}(\Theta)=n$. But in (and only in) such a case, there would be $\lambda=\sum_{\theta \in \Theta} c_{\theta} \delta_{\theta} \in \mathcal{M}(\Theta)$, which is orthogonal to $H$, i.e.,

$$
\sum_{\theta \in \Theta} c_{\theta} p(\theta)=0, \quad \text { all } \quad p \in H
$$

Defining $f:=\sum_{\theta \in \Theta} c_{\theta} e_{\theta}$, we obtain an exponential $f \in E_{n}(\Omega)$ such that $p(D) f(0)$ $=\lambda p=0$, for every $p \in H$. For $p \in H \cap \Pi_{k}^{0}$, we have

$$
0=p(D) f(0)=p(D)(\bar{k}(f))(0)=(\bar{k}(f)(D)) p(0)=(\bar{k}(f)(D)) p .
$$

Therefore, the condition $p(D) f(0)=0$, all $p \in H$, is equivalent to $\bar{k}(f)(D)$ annihilating $H \cap \Pi_{k}^{0}$ for every $k$.

The principal result of this section is as follows:

Theorem 6.3. Let $K \subset \mathbb{Z}_{+}$, and let $n$ be a positive integer. Then $K$ is not total of order order $n$ for $S^{m-1}$, if and only if there exists $f \in E_{n}\left(S^{m-1}\right)$ for which $\bar{K}(f) \in \delta \mathbf{A}$. 
In fact, the proof of Theorem 6.3 is more informative than its statement: given $\Theta \subset S^{m-1}$, the proof shows that the matrix $A_{g, \Theta}$ is singular if and only if there exists an exponential $f \in E(\Theta)$ that satisfies $\overline{K_{m, g}}(f) \in \delta \mathbf{A}$.

Proof of Theorem 6.3. By Theorem 3.1 (and in view of Definitions 2.9 and 6.1) the required property of $K$ is equivalent to $\mathcal{H}_{K}$ being total of order $n$ for $S^{m-1}$. Therefore, in view of Theorem 6.2, we need to prove that the condition stated in the present theorem is equivalent to the following: "There exists $f \in E_{n}\left(S^{m-1}\right)$ such that, for every $k \in K,(\bar{k}(f))(D)$ annihilates $\mathcal{H}_{K} \cap \Pi_{k}^{0}=\mathcal{H}_{k}^{0}$." Since $\mathcal{H}_{k}^{0}$ is the kernel in $\Pi_{k}^{0}$ of the Laplacian $\delta(D)$, the last condition is equivalent to the divisibility of $\bar{k}(f)$ by $\delta$.

The following sufficient condition is derived from the characterization in Theorem 6.3 .

Theorem 6.4. Given $\Theta \subset S^{m-1}$, define

$$
\sigma(\Theta):=\min \left\{\# \Theta^{\prime} \subset \Theta: \operatorname{span}\left(\Theta \backslash \Theta^{\prime}\right) \neq \mathbb{R}^{m}\right\} .
$$

Let $j$ be the minimal integer that satisfies $\left(\begin{array}{c}j+m-2 \\ m-1\end{array}\right)>\sigma(\Theta)$. Let $g$ be a positive definite function for $S^{m-1}$, and assume that the set $\left\{k \in K_{m, g}: k \geq(\# \Theta) / 2\right\}$ contains $j$ consecutive even integers as well as $j$ consecutive odd integers. Then $A_{g, \Theta}$ is invertible.

We divide the proof of Theorem 6.4 into a sequence of several lemmas; some of them might appear to be of independent interest. Note that $\left(\begin{array}{c}j+m-2 \\ m-1\end{array}\right)=\operatorname{dim} \Pi_{j-1}^{0}$.

Lemma 6.5. The operator $\delta(D)$ induces an isomorphism between $\delta^{n+1} \Pi$ and $\delta^{n} \Pi$, $n=0,1, \ldots$.

Proof. First, we recall that $\Pi$ is the direct sum of the spaces $\left(\delta^{k} \mathcal{H}\right)_{k \in \mathbb{Z}_{+}}$(A quick proof of that would go as follows. Since $\mathcal{H}$ is the kernel in $\Pi$ of the homogeneous differential operator $\delta(D)$, $\Pi$ is the direct sum of $\mathcal{H}$ and the principal ideal $\delta \Pi$ :

$$
\Pi=\mathcal{H} \oplus \delta \Pi \text {. }
$$

Multiplying the above equation by $\delta^{k}$, and using induction, we obtain

$$
\left.\Pi=\left(\sum_{j=0}^{k-1} \delta^{j} \mathcal{H}\right) \oplus \delta^{k} \Pi=\left(\sum_{j=0}^{k} \delta^{j} \mathcal{H}\right) \oplus \delta^{k+1} \Pi .\right)
$$

Next, one checks directly that, for each $k \in \mathbb{Z}_{+}$, there exists a constant $c_{n, k}$ such that the operator $p \mapsto c_{n, k}(\delta(D) p) \delta$ is the identity on $\delta^{n+1} \mathcal{H}_{k}^{0}$. This implies that $\delta(D)$ maps $\delta^{n+1} \mathcal{H}$ one-to-one onto $\delta^{n} \mathcal{H}$.

Finally, the decomposition result asserted in the first paragraph of the proof allows us to write

$$
\delta^{n+1} \Pi=\bigoplus_{k \geq n+1} \delta^{k} \mathcal{H}
$$

The desired result then follows from an application of $\delta(D)$ to both sides of the last equality, and invoking the isomorphism assertion from the second paragraph of the proof. 
Lemma 6.6. Assume that $f \in \mathbf{A}$ and satisfies $\delta(D) f=f$. Assume further that for some $k, n \in \mathbb{Z}_{+}$,

$$
(\overline{k+2 j})(f) \in \delta \Pi, \quad j=0, \ldots, n .
$$

Then,

$$
(\overline{k+2 n})(f) \in \delta^{n+1} \Pi
$$

Proof. By induction on $n$. If $n=0$, the claim in (6.8) is assumed in equation (6.7). Assume thus that the claim is valid for $n-1 \geq 0$. Since $\delta(D) f=f$, we have that $\delta(D)((\overline{k+2 n})(f))=(\overline{k+2 n-2})(f)$, hence by the induction hypothesis

$$
\delta(D)((\overline{k+2 n})(f)) \in \delta^{n} \Pi .
$$

Invoking Lemma 6.5, we conclude that

$$
(\overline{k+2 n})(f) \in \mathcal{H}+\delta^{n+1} \Pi .
$$

The result then follows from the assumption that $(\overline{k+2 n})(f)$ is divisible by $\delta$ (and the fact that no polynomial in $\mathcal{H} \backslash 0$ is divisible by $\delta$ ).

Proof of Theorem 6.4. Let $\Theta \subset S^{m-1}$ of cardinality $n$ be given, and let $f \in E(\Theta)$. By Theorem 6.3 (more precisely, by the statement made in the paragraph that follows Theorem 6.3), we need to show that $\bar{K}(f)$ is not divisible by $\delta$. Assume, to the contrary, that $\bar{K}(f)$ is divisible by $\delta$. We first note that, for some $k \leq n / 2$, say, $k_{0}$, one has $\overline{k_{0}}(f) \neq 0$. (Indeed, by the definition (4.5) of $\Pi_{\Theta}$, if $\bar{k}(f)=0$ for all $k \leq n / 2$, then $\Pi_{\Theta}$ must contain a polynomial of degree $>n / 2$, in contradiction with Lemma 4.4). Since $f$, as any function in $E\left(S^{m-1}\right)$, satisfies $\delta(D) f=f$, one concludes from the fact that $\overline{k_{0}}(f) \neq 0$, that $\bar{i}(f) \neq 0$, for every $i \in k_{0}+2 \mathbb{Z}_{+}$. Because $k_{0} \leq n / 2$, our assumptions on $K$ imply that there exist $j$ integers $k, k-$ $2, k-4, \ldots, k-2 j+2$ in $K$, that further satisfy $k-2 j+2-k_{0} \in 2 \mathbb{Z}_{+}$. In particular, each integer $i$ in this progression satisfies the relation $\bar{i}(f) \neq 0$. On the other hand, each of these integers lies in $K$, and since $\bar{K}(f) \in \delta \Pi$, Lemma 6.6 implies that

$$
\bar{k}(f) \in \delta^{j} \Pi \text {. }
$$

As the rest of the proof will establish, this last conclusion contradicts the fact that $f \in E(\Theta)$.

Since $f$ is a linear combination of $\left\{e_{\theta}\right\}_{\theta \in \Theta}$, we have that

$$
\bar{k}(f)=\sum_{\theta \in T} c_{\theta}(\theta \cdot)^{k}
$$

with $T \subset \Theta$, and with any $\theta, \theta^{\prime} \in T$ being linearly independent. Here,

$$
(\theta \cdot)^{k}: x \mapsto(\theta \cdot x)^{k} .
$$

Let $T^{\prime}$ be a nonspanning set in $T$ of maximal cardinality. Since $\operatorname{dim} \Pi_{j-1}^{0}>\sigma(\Theta) \geq$ $\#\left(T \backslash T^{\prime}\right)$, there exists a nontrivial homogeneous polynomial $p_{1} \in \Pi_{j-1}^{0}$ that vanishes on $T \backslash T^{\prime}$. Writing $p_{1}$ in the form $p_{1}=\delta^{i} p$ with $p \notin \delta \Pi$ and $i \geq 0$, we conclude that $p$ is a homogeneous polynomial of degree $r<j$ which vanishes on $T \backslash T^{\prime}$. An application of $p(D)$ to $\bar{k}(f)$ yields a linear combination of the form

$$
q=\sum_{\theta \in T^{\prime}} a_{\theta}(\theta \cdot)^{k-r} .
$$

Since $T^{\prime}$ does not span, the above polynomial is of less than $m$ variables. 
On the other hand, $\bar{k}(f)=\delta^{s} P$, for some $P \in \Pi \backslash(\delta \Pi), s \geq j$. Therefore, for some $c \neq 0$,

$$
p(D)(\bar{k}(f))=p(D)\left(\delta^{s} P\right)=c p \delta^{s-r} P+\delta^{s-r+1} P_{1} .
$$

Since $c p P$ is nonzero and is not divisible by $\delta$, we conclude that $p(D)(\bar{k}(f))$ is a nonzero polynomial in $\delta \Pi$, hence, in particular, cannot be of less than $m$ variables, and we thus have reached the desired contradiction.

Since $\sigma(\Theta) \leq n-m+1$ for any $\Theta \subset \mathbb{R}^{m}$ of cardinality $n$, we obtain the following corollary:

Corollary 6.9. Let $K \subset \mathbb{Z}_{+}$, and $n$ be a positive integer. Then $K$ is total of order $n$ on $S^{m-1}$, if, with $j$ the minimal integer that satisfies $\left(\begin{array}{c}j+m-2 \\ m-1\end{array}\right)>n-m+1$, there are $j$ consecutive even integers and $j$ consecutive odd integers in the set $\{k \geq$ $n / 2\} \cap K$.

In particular, if $K$ contains arbitrarily long sequences of consecutive even and of consecutive odd integers, then $K$ induces strict positive definiteness (of all orders) on $S^{m-1}$, for every $m \geq 2$.

Note that, in Theorem 6.4, $\sigma(\Theta)=1$ whenever $\Theta$ does not span. Thus, the theorem implies that, if we sum two Gegenbauer polynomials associated with $S^{m-1}$, one of even degree and one of odd degree, we get a function $g$ that is strictly positive definite of order $n$ for $S^{l-1}, l<m$, provided that the degree of the above-mentioned polynomials is $\geq n / 2$. In particular, we obtain that $g$ is strictly positive definite of all orders on $S^{l-1}$, if it is positive definite of order $m>l$, and $K_{m, g}$ contains infinitely many odd and infinitely many even integers (compare with Corollary 4.4 of [12]). Actually, such observations already follow from Theorem 4.1: the known interrelations among Gegenbauer polynomials of different types (cf. [1]) imply that, if $K_{m, g}$ contains one odd integer $\geq k$, and one even integer $\geq k$, then $K_{l, g}$ contains all integers $\leq k$.

\section{ACKNOWLEDGMENT}

This work was partially supported by NSF (grants DMS-9102857 and DMS9224748) and by ARO (contracts DAAL03-90-G-0090 and DAAH04-95-1-0089). Also, we thank Professor E.W. Cheney and the referee for their suggestions; material from an unpublished note of Professor Cheney is used in $\S 5$.

\section{REFERENCES}

1. R. Askey and J. Fritch, Integral representations for Jacobi polynomials, and some applications, J. Math. Anal. Appl. 26 (1969), 411-437. MR 38:6128

2. C. de Boor and A. Ron, On multivariate polynomial interpolation, Constructive Approximation 6 (1990), 287-302. MR 91c: 41005

3. Computational aspects of polynomial interpolation in several variables, Mathematics of Computation 58 (1992), 705-727. MR 92i:65022

4. $\quad$ The least solution of the multivariate polynomial interpolation, Math. Z. 210 (1992), 347-378. MR 93f: 41002

5. E. W. Cheney and Yuan $\mathrm{Xu}, A$ set of research problems in approximation theory, Topics in Polynomials of One and Several Variables and Their Applications (Th. M. Rassias, H. M. Srivastava, and A. Yanushauskas, eds., eds.), World Scientific, River Edge, NJ, 1993. MR 95c:41001 
6. N. Dyn, Interpolation and approximation by radial and related functions, Approximation VI (C. K. Chui, L. L. Schumaker, and J. D. Ward, eds.), vol. I, 1989, Academic Press, pp. 211-234. MR 92d:41002

7. W. A. Light and E. W. Cheney, Interpolation by periodic radial basis functions, J. Math. Anal. Appl. 168 (1992), 111-130. MR 93f:41039

8. C. A. Micchelli, Interpolation of scattered data: distance matrices and conditionally positive definite functions, Constr. Approx. 2 (1986), 11-22. MR 88d:65016

9. V. A. Menegatto, Strictly positive definite functions on spheres, University of Texas at Austin, 1992.

10. Strictly positive definite kernels on circle, Rocky Mountain J. Math. 25 (1995), 11491163. CMP 96:03

11. Strictly positive definite kernels on the Hilbert sphere, Appl. Anal. 55 (1994), 81-101.

12. F. J. Narcowich, Generalized Hermite interpolation and positive definite kernels on a Riemannian manifold, J. Math. Anal. Appl. 190 (1995), 165-193. MR 96c:41009

13. F. J. Narcowich and J. D. Ward, Norms of inverses and condition numbers of matrices associated with scattered data, J. Approx. Theory 64 (1991), 69-94. MR 92b:65017

14. - Norm estimates for the inverses of a general class of scattered data radial function interpolation matrices, J. Approx. Theory 69 (1992), 84-109. MR 93c:41005

15. E. Quak, N. Sivakumar, and J. D. Ward, Least squares approximation by radial functions, SIAM J. Math. Anal. 24 (1993), 1043-1066. MR 94g:41059

16. A. Ron and X. Sun, Strictly positive definite functions on spheres, CMS TR 94-6, University of Wisconsin - Madison, February 1994.

17. I. J. Schoenberg, Metric spaces and completely monotone functions, Ann. Math. 39 (1938), 811-841.

18. _ Positive definite functions on spheres, Duke Math. J. 9 (1942), 96-108. MR 3:232c

19. E. M. Stein and G. Weiss, Introduction to Fourier Analysis on Euclidean Spaces, Princeton University Press, Princeton, NJ, 1971. MR 46:4102

20. N. Sivakumar and J. D. Ward, On the least squares fit by radial functions to multidimensional scattered data, Numer. Math. 65 (1993), 219-243. MR 94d:41006

21. X. Sun, The fundamentality of translates of a continuous function on spheres, Numerical Algorithms 8 (1994), 131-134. MR 96a:41005

22. Gabor Szegö, Orthogonal Polynomials, Amer. Math. Colloq. Publ., Amer. Math. Soc., Providence, RI, 1959. MR 21:5029

23. Yuan Xu and E. W. Cheney, Strictly positive definite functions on spheres, Proc. Amer. Math. Soc. 116 (1992), 977-981. MR 93b:43005

Department of Computer Science, University of Wisconsin-Madison, Madison, WisCONSIN 53706

Department of Mathematics, Southwest Missouri State University, Springfield, MisSOURI 65804 